\title{
THE GENERALIZED PELLIAN EQUATION
}

\author{
BY \\ LEON BERNSTEIN
}

1. Summary of results. It is known from number theory that the rational integral solutions of the Diophantic equation in two unknowns of second degree, viz.

$$
x^{2}-m y^{2}= \pm 1,
$$

where $m$ is not the square of a rational integer, are obtained from the numerator and denominator of the convergents in the development of $m^{1 / 2}$ as a periodic continued fraction. (1.1) is known as the Pellian equation.

Let $m$ be a natural number which is not the $n$th power of a rational integer. In the present paper we solve, in rational integers, the Diophantic equation in $n$ unknowns of degree $n \geqq 2$

$$
D\left(m ; x_{1}, \ldots, x_{n}\right)= \pm 1,
$$

with the notation

$$
D\left(m ; x_{1}, \ldots, x_{n}\right)=\left|\begin{array}{crrrrr}
x_{1} & x_{2} & x_{3} & \cdots & x_{n-1} & x_{n} \\
m x_{n} & x_{1} & x_{2} & \cdots & x_{n-2} & x_{n-1} \\
m x_{n-1} & m x_{n} & x_{1} & \cdots & x_{n-3} & x_{n-2} \\
\cdot \cdot & \cdot & \cdot & \cdot & \cdot & \cdot \\
m x_{3} & m x_{4} & m x_{5} & \cdots & x_{1} & x_{2} \\
m x_{2} & m x_{3} & m x_{4} & \cdots & m x_{n} & x_{1}
\end{array}\right| .
$$

(1.2) is also solved by means of the convergents appearing in the Jacobi-Perron algorithm whose theory was developed by the author in a series of previous papers [1 (a)-(q)]. For $n=2$ (1.2) becomes the Pellian equation, viz.

$$
\left|\begin{array}{rr}
x_{1} & x_{2} \\
m x_{2} & x_{1}
\end{array}\right|=x_{1}^{2}-m x_{2}^{2}= \pm 1 .
$$

For this reason (1.2) is called the generalized Pellian equation. It is remarkable that by means of the periodic Jacobi-Perron algorithm, which solves (1.2) it is possible to find an infinite system of integral solutions for a generalized Pellian equation of a much broader type. Let

$$
m=D^{n}+d ; D, d, n \text { natural numbers; } n \geqq 2 ; d \mid D .
$$

Received by the editors June 5, 1966. 
Then, in addition to (1.2) an infinite system of solutions is found for the Diophantic equation

$$
D\left(m ; x_{1}, \ldots, x_{n}\right)=(-1)^{k(n-1)} d^{k} \quad(k=1, \ldots, n-1) .
$$

We shall call (1.2) the generalized Pellian equation of zero-type, and (1.5) the generalized Pellian equation of $k$-type. Further let

$$
m=D^{n}-d ; D, d, n \text { natural numbers; } n \geqq 2 ; d \mid D \text {. }
$$

For such $m$ we shall call (1.2) the generalized Pellian equation of minus zero-type and shall use the notation \pm zero-type for $m=D^{n} \pm d$.

Connections between the integral solutions of the generalized Pellian equation of \pm zero-type and the structure of units of the field $Q\left(m^{1 / n}\right)$ are revealed. I want to express my gratitude to the referee of this paper for having drawn my attention to this important fact and for the many valuable remarks and hints which helped to improve my results.

2. Units and Diophantic equations. Let $\boldsymbol{m}$ be a positive rational, $n$ a natural number $\geqq 2$, and write

$$
w=m^{1 / n}
$$

Let $w$ be a positive real $n$th degree irrational. In the field $Q(w)$ every number $X$ has the form

$$
X=x_{1}+x_{2} w+x_{3} w^{2}+\cdots+x_{n} w^{n-1} \quad\left(x_{1}, \ldots, x_{n} \text { rationals }\right) .
$$

We shall prove that the norm of $X$ is expressed through the components $x_{i}$ $(i=1, \ldots, n)$ by the formula

$$
N(X)=D\left(m ; x_{1}, \ldots, x_{n}\right) .
$$

The conjugate roots of $w^{n}=m$ are

$$
w_{k}=u^{k} w ; \quad u=\exp [2 \pi k i / n] \quad(k=0,1, \ldots, n-1),
$$

and $N(X)$ has the form

$$
N(X)=\prod_{k=0}^{n-1}\left(x_{1}+x_{2} w_{k}+x_{3} w_{k}^{2}+\cdots+x_{n} w_{k}^{n-1}\right)
$$

Since $N(X)$ is a rational number and $w_{k}$ an $n$th degree irrational, only integral powers of $w_{k}^{n}$ can appear on the right-hand side of (2.5). Since

$$
\begin{gathered}
\prod_{k=0}^{n-1} u^{s k}=(-1)^{s(n-1)}, \\
N(X)=\sum_{s=0}^{n-1}(-1)^{s(n-1)} m^{s} x_{s+1}^{n}+\sum x_{1}^{j_{1}} x_{2}^{j_{2}} \ldots x_{n}^{j_{n}}, \quad 0 \leqq j_{1}, \ldots, j_{n}<n .
\end{gathered}
$$


Thus the powers $x_{i}^{n}(i=1, \ldots, n)$ do not appear under the second sigma sign, and $x_{1}^{n}$ has the coefficient 1 . Since $x_{1}+x_{2} w+x_{3} w^{2}+\cdots+x_{n} w^{n-1}$ is in $Q(w)$, so is $\prod_{k=1}^{n-1}\left(x_{1}+x_{2} w_{k}+x_{3} w_{k}^{2}+\cdots+x_{n} w_{k}^{n-1}\right)$, so that

$$
N(X)=\left(x_{1}+x_{2} w+\cdots+x_{n} w^{n-1}\right)\left(y_{1}+y_{2} w+\cdots+y_{n} w^{n-1}\right)
$$

$\left(y_{1}, \ldots, y_{n}\right.$ rationals $)$.

On the right-hand side of (2.7) all the powers of $w$ but $w^{n}$ must vanish. This gives the following system of $n$ linear equations in the $y_{i}(i=1, \ldots, n)$

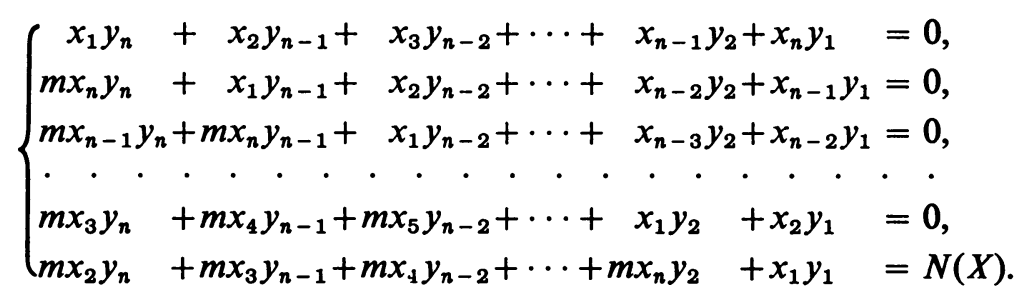

The solution of (2.8) (under the presumption that $X$ is not identically zero) is given by the formula

$$
y_{k}=\frac{N(X) A_{n, n+1-k}\left(m ; x_{1}, \ldots, x_{n}\right)}{D\left(m ; x_{1}, \ldots, x_{n}\right)} \quad(k=1, \ldots, n) .
$$

Here $A_{n, n+1-k}\left(m ; x_{1}, \ldots, x_{n}\right)$ denotes the algebraic component of the element $a_{n, n+1-k}$ in the determinant $D\left(m ; x_{1}, \ldots, x_{n}\right)=\left|a_{i j}\right|$. From (2.7) we obtain, in view of (2.6), that the highest power of $x_{1}$ in the expression of $y_{1}$ is $x_{1}^{n-1}$ with coefficient 1 ; the highest power of $x_{1}$ in the development of $A_{n, n}\left(m ; x_{1}, \ldots, x_{n}\right)$ is also $x_{1}^{n-1}$ with coefficient 1 . Thus we obtain from (2.9) $N(X) / D\left(m ; x_{1}, \ldots, x_{n}\right)=1$ which proves (2.3). Formula (2.9) takes the form

$$
y_{k}=A_{n, n+1-k}\left(m ; x_{1}, \ldots, x_{n}\right) \quad(k=1, \ldots, n) .
$$

Now let $X$ be in the ring $R(w)$ of the field $Q(w)$. Then the $x_{i}(i=1, \ldots, n)$ are rational integers. Let $X_{e}$ be a unit in $R(w)$ of the field $Q(w)$. A known theorem about the norm of a unit, combined with formula (2.3) states

THEOREM 1. Let $m$ be a natural number and $w=m^{1 / n}$ a real $n$th degree irrational. $A$ necessary and sufficient condition that the number $X_{e}=x_{1}+x_{2} w+x_{3} w^{2}+\cdots$ $+x_{n} w^{n-1}$ in the ring $R(w)$ be a unit of the field $Q(w)$ is the solution of the Diophantic equation $D\left(m ; x_{1}, \ldots, x_{n}\right)= \pm 1$ in rational integers $x_{i}(i=1, \ldots, n)$.

With $X_{e}$ the number $X_{e}^{-1}=Y_{e}$ is also a unit in $Q(w)$. Combining (2.7) and (2.10) we obtain from Theorem 1 .

Corollary 1. If the $n$-tuple $\left(x_{1}, x_{2}, \ldots, x_{n}\right)$ is a solution of $D\left(m ; x_{1}, \ldots, x_{n}\right)$ $= \pm 1$, then the $n$-tuple

$$
\left(y_{1}, y_{2}, \ldots, y_{n}\right), \quad y_{k}=A_{n, n+1-k}\left(m ; y_{1}, \ldots, y_{n}\right)
$$

is another solution of that equation. 
Following Dirichlet's fundamental theorem about units of a field the number of basic units of $Q\left(m^{1 / n}\right), m$ a positive rational, equals [n/2]. Here [x] denotes, as customary, the greatest rational integer not exceeding $x$. Thus every unit supplies, through its positive and negative powers, two infinite systems of solutions of $D\left(m ; x_{1}, \ldots, x_{n}\right)= \pm 1$ in rational integers. In this paper we shall find a unit in $R(w)$. Whether the other independent units of the field $Q(w)$ are in the ring $R(w)$ is an open and challenging question.

3. The algorithm of Jacobi-Perron. This is defined in the following way: let

$$
a_{1}^{(0)}, a_{2}^{(0)}, \ldots, a_{n-1}^{(0)} \quad(n \geqq 2)
$$

be a set of $n-1$ real numbers; from it new sets of $n-1$ numbers each are obtained by the following rule:

$$
\begin{aligned}
a_{n-1}^{(v+1)} & =\frac{1}{a_{1}^{(v)}-b_{1}^{(v)}}, \\
a_{k}^{(v+1)} & =\frac{a_{k+1}^{(v)}-b_{k+1}^{(v)}}{a_{1}^{(v)}-b_{1}^{(v)}} \quad(k=1, \ldots, n-2), \\
b_{i}^{(v)} & =\left[a_{i}^{(v)}\right] \quad(i=1, \ldots, n-1 ; v=0,1, \ldots) .
\end{aligned}
$$

The algorithm is called periodic, if there exist nonnegative integers $s$ and natural numbers $t$ such that

$$
a_{i}^{(t+v)}=a_{i}^{(v)} \quad(i=1, \ldots, n-1 ; v=s, s+1, \ldots) .
$$

Let

$$
\min s=S ; \quad \min t=T .
$$

Then the $S$ sets

$$
a_{1}^{(v)}, a_{2}^{(v)}, \ldots, a_{n-1}^{(v)} \quad(v=0,1, \ldots, S-1)
$$

are called the pre-period of the algorithm, $S$ is called its length; the $T$ sets

$$
a_{1}^{(v)}, a_{2}^{(v)}, \ldots, a_{n-1}^{(v)} \quad(v=S, S+1, \ldots, S+T-1)
$$

are called the period of the algorithm, $T$ is called its length. The sum $S+T$ is called the length of the algorithm; if $S=0$, the algorithm is called purely periodic.

For $n=2$ the algorithm becomes the Euclidean algorithm; for $n=4$ it was first introduced by Jacobi [2]; its theory for any $n \geqq 2$ was masterly developed by Perron [3]. If the rational integers $A_{i}^{(v)}$ are defined by the recursion formulas in the following way

$$
\begin{aligned}
A_{i}^{(i)} & =1 ; A_{i}^{(v)}=0 \quad(i \neq v ; i, v=0,1, \ldots, n-1) \\
A_{i}^{(v+n)} & =A_{i}^{(v)}+\sum_{j=1}^{n-1} b_{j}^{(v)} A_{i}^{(v+j)} \quad(i=0, \ldots, n-1 ; v=0,1, \ldots),
\end{aligned}
$$


then, as has been proved by the author [1(m)] the formulas hold:

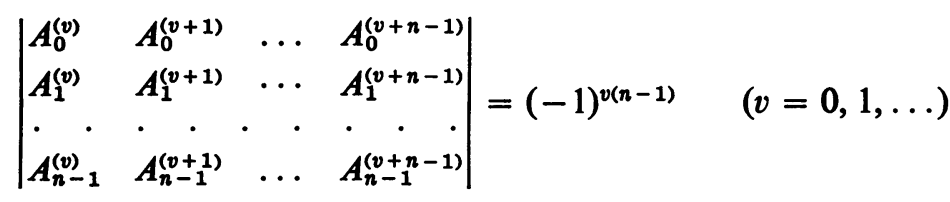

$$
\begin{aligned}
& a_{i}^{(0)}=\frac{A_{i}^{(v)}+\sum_{j=1}^{n-1} a_{j}^{(v)} A_{i}^{(v+j)}}{A_{0}^{(v)}+\sum_{j=1}^{n-1} a_{j}^{(v)} A_{0}^{(v+j)}} \quad(i=1, \ldots, n-1 ; v=0,1, \ldots) .
\end{aligned}
$$

The key to the solution of the Diophantic equations (1.2) and (1.5) depends upon the following theorem of the author [1(a)].

THEOREM. Write

$$
w=\left(D^{n}+d\right)^{1 / n}, \quad D, d, n \text { natural numbers; } d \mid D ; D \geqq(n-2) d ; n \geqq 2 .
$$

The Jacobi-Perron algorithm of the $n-1$ numbers

$$
w, w^{2}, \ldots, w^{n-1}
$$

is periodic and the lengths of the period and preperiod are respectively

$$
\begin{aligned}
& T=n ; S=n-1 \quad \text { for } d>1 ; \\
& T=1 ; S=n-1 \quad \text { for } d=1 .
\end{aligned}
$$

In case $d>1$ the $a_{i}^{(n-1+v)}(i=1, \ldots, n-1 ; v=0,1, \ldots, n-1)$ have the form

$$
\begin{aligned}
& a_{i}^{(n-1)} \quad=d^{-1} \sum_{j=0}^{i}\left(\begin{array}{c}
n-1-i+j \\
j
\end{array}\right) w^{i-j} D^{j} \quad(i=1, \ldots, n-1), \\
& a_{i}^{(n)} \quad=\quad \sum_{j=0}^{i}\left(\begin{array}{c}
n-1-i+j \\
j
\end{array}\right) w^{i-j} D^{j} \quad(i=1, \ldots, n-1), \\
& \left\{\begin{aligned}
a_{i}^{(n+k)}= & \sum_{j=0}^{i}\left(\begin{array}{c}
n-1-i+j \\
j
\end{array}\right) w^{i-j} D^{j} \quad(i=1, \ldots, n-1-k), \\
a_{n-1-k+s}^{(n+k)}=d^{-1} \sum_{j=0}^{n-1-k+s}\left(\begin{array}{c}
k-s+j \\
j
\end{array}\right) w^{n-1-k+s-j} D^{j} & (s=1, \ldots, k), k=1, \ldots, n-2 .
\end{aligned}\right.
\end{aligned}
$$

In case $d=1$ the $a_{i}^{(n-1)}$ have the form as the $a_{i}^{(n)}$ in (3.13). The very simple form of the period is stated explicitly in the original form of this theorem.

4. The generalized Pellian equation of zero-type. In this chapter we shall solve the generalized Pellian equation of \pm zero-type and prove, to this end, 
Theorem 2. An infinite system of solutions of the generalized Pellian equation of + zero-type, viz. $D\left(m ; x_{1}, \ldots, x_{n}\right)=+1$ is given by the formula

$$
x_{k}=\sum_{j=0}^{n-k}\left(\begin{array}{c}
n-k \\
j
\end{array}\right) D^{s} A_{0}^{(s n+k-1+j)} \quad(k=1, \ldots, n ; s=1,2, \ldots) .
$$

The numbers $A_{0}^{(s n+k-1+j)}$ as defined by (3.7) are obtained from the periodic JacobiPerron algorithm of the numbers $w, w^{2}, \ldots, w^{n-1} ; w, D, d, n$ have the meaning of (3.10) and $d>1$.

For $d=1$ the generalized Pellian equation

$$
D\left(m ; x_{1}, \ldots, x_{n}\right)=(-1)^{s(n-1)} \quad(s=0,1, \ldots)
$$

has an infinite system of solutions given by the formula

$$
x_{k}=\sum_{j=0}^{n-k}\left(\begin{array}{c}
n-k \\
j
\end{array}\right) D^{s} A_{0}^{(n+s+k-1+j)} \quad(k=1, \ldots, n ; s=0,1, \ldots) .
$$

Proof. We shall introduce the notation

$$
\begin{array}{ll}
x_{k}^{(i)}=\sum_{j=0}^{n-k}\left(\begin{array}{c}
n-k \\
j
\end{array}\right) D^{j} A_{i}^{(s n+k-1+j)} & (k-1, i=0, \ldots, n-1 ; s=1,2, \ldots), \\
x_{k}^{(0)}=x_{k} & (k=1, \ldots, n) .
\end{array}
$$

We shall make use of formula (3.8). For $v=s n(s=1,2, \ldots)$ we obtain, since $(-1)^{s n(n-1)}=1$,

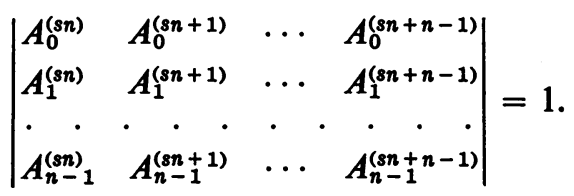

If we add to the $k$ th column vector of the determinant (4.5) successively the $D\left(C_{n-k, 1}\right)$ multiple of the $k+1$ th vector, then the $D^{2}\left(C_{n-k, 1}\right)$ multiple of the $k+2$ th vector, $\ldots$, and finally the $D^{n-k}\left(C_{n-k, n-k}\right)$ multiple of the $k+(n-k)$ $=n$th vector $(k=1, \ldots, n-1)$, the determinant (4.5) with the notation of (4.4) takes the form

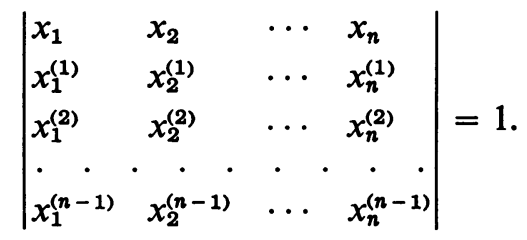

We shall now make use of formula (3.9) and obtain for $v=s n$

$$
a_{i}^{(0)}=\frac{A_{i}^{(s n)}+\sum_{j=1}^{n-1} a_{j}^{(s n)} A_{i}^{(s n+j)}}{A_{0}^{(s n)}+\sum_{j=1}^{n-1} a_{j}^{(s n)} A_{0}^{(s n+j)}} \quad(i=1, \ldots, n-1) .
$$


But since the period of the Jacobi-Perron algorithm of the numbers $w, w^{2}, \ldots, w^{n-1}$ has the length $n$ for $d>1$, we obtain $a_{j}^{(s n)}=a_{j}^{(n)}(j=1, \ldots, n-1)$ and formula (4.7) takes the form

$$
a_{i}^{(0)}=\frac{A_{i}^{(s n)}+\sum_{j=1}^{n-1} a_{j}^{(n)} A_{i}^{(s n+j)}}{A_{0}^{(s n)}+\sum_{j=1}^{n-1} a_{j}^{(n)} A_{0}^{(s n+j)}} \quad(i=1, \ldots, n-1)
$$

Substituting in (4.8) the values from (3.13), viz.

$$
a_{i}^{(0)}=w^{i} ; \quad a_{i}^{(n)}=\sum_{j=0}^{i}\left(\begin{array}{c}
n-1-i+j \\
j
\end{array}\right) w^{i-j} D^{j} \quad(i=1, \ldots, n-1),
$$

we obtain

$$
w^{i}=\frac{A_{i}^{(s n)}+\sum_{j=1}^{n-1}\left(\sum_{u=0}^{j}\left(\begin{array}{c}
n-1-j+u \\
u
\end{array}\right) w^{j-u} D^{u}\right) A_{i}^{(s n+j)}}{A_{0}^{(s n)}+\sum_{j=1}^{n-1}\left(\sum_{u=0}^{j}\left(\begin{array}{c}
n-1-j+u \\
u
\end{array}\right) w^{j-u} D^{u}\right) A_{0}^{(s n+j)}} \quad(i=1, \ldots, n-1)
$$

Arranging the numerator and denominator of (4.9) in powers of $w$, we obtain easily, with the notation of (4.4),

$$
w^{i}=\frac{x_{1}^{(i)}+x_{2}^{(i)} w+x_{3}^{(i)} w^{2}+\cdots+x_{n}^{(i)} w^{n-1}}{x_{1}+x_{2} w+x_{3} w^{2}+\cdots+x_{n} w^{n-1}} \quad(i=1, \ldots, n-1)
$$

If we multiply in (4.10) $w^{i}$ by the denominator and take in account that $w^{n}=D^{n}+d=m$, we obtain, arranging this product in powers of $w^{k}, k \leqq n-1$,

$$
\begin{aligned}
x_{1}^{(i)} & +x_{2}^{(i)} w+x_{3}^{(i)} w^{2}+\cdots+x_{n}^{(i)} w^{n-1} \\
& =x_{1} w^{i}+x_{2} w^{i+1}+\cdots+x_{n-i} w^{n-1}+m\left(x_{n-i+1}+x_{n-i+2} w+\cdots+x_{n} w^{i-1}\right) .
\end{aligned}
$$

Since, as was proved by the author in previous papers [1(g), (h), (m)] w is an $n$th degree irrational, the coefficients of equal powers of $w$ on both sides of the equation (4.11) must be identical, which gives, for each $i$, the following $n$ equations

$$
\begin{array}{cc}
x_{s}^{(i)}=m x_{n-i+s} & (s=1, \ldots, i), \\
x_{i+t}^{(i)}=x_{t} \quad & (t=1, \ldots, n-i), \\
& i=1, \ldots, n-1 .
\end{array}
$$

Substituting the values of (4.12) in (4.6) we obtain the determinant (1.3) which proves Theorem 2, for $d>1$. 
For $d=1$ the period is of length 1 and $a_{i}^{(n+s)}=a_{i}^{(n)},(i=1, \ldots, n-1 ; s=0,1, \ldots)$ so that formula (3.9) takes the form, for $v=n+s$,

$$
\begin{aligned}
a_{i}^{(0)}= & \frac{A_{i}^{(n+s)}+\sum_{j=1}^{n-1} a_{j}^{(n+s)} A_{i}^{(n+s+j)}}{A_{0}^{(n+s)}+\sum_{j=1}^{n-1} a_{j}^{(n+s)} A_{0}^{(n+s+j)}} \\
= & \frac{A_{i}^{(n+s)}+\sum_{j=1}^{n-1} a_{j}^{(n)} A_{i}^{(n+s+j)}}{A_{0}^{(n+s)}+\sum_{j=1}^{n-1} a_{j}^{(n)} A_{0}^{(n+s+j)}} \quad(s=0,1, \ldots) .
\end{aligned}
$$

With the value $v=n+s$ the determinant (3.8) equals $(-1)^{(n+s)(n-1)}=(-1)^{s(n-1)}$. We then proceed exactly as in the case of $d>1$ with the only exception that in the determinant (4.5) we have to write $n+s$ instead of $n s$ to obtain formula (4.3) for the solution of the equation (4.2). This completes the proof of Theorem 2.

It should be noted that formula (4.1) is also valid for $s=0$. In this case the generalized Pellian equation of + zero-type has the solution:

$$
x_{1}=1 ; \quad x_{2}=x_{3}=\cdots=x_{n}=0 .
$$

Recent results of the author $[1(\mathrm{~m})]$ have shown that the restriction $D \geqq d(n-2)$ can be dropped; only $d \mid D$ is necessary.

EXAMPLE. $n=3 ; D=2 ; d=1 ; m=2^{3}+1=9$. The generalized Pellian equation takes the form, since $(-1)^{s(n-1)}=(-1)^{2 s}=1$,

$$
\begin{aligned}
\left|\begin{array}{rrr}
x_{1} & x_{2} & x_{3} \\
m x_{3} & x_{1} & x_{2} \\
m x_{2} & m x_{3} & x_{1}
\end{array}\right| & =N\left(x_{1}+x_{2} m^{1 / 3}+x_{3} m^{2 / 3}\right) \\
& =x_{1}^{3}+m x_{2}^{3}+m^{2} x_{3}^{3}-3 m x_{1} x_{2} x_{3}=1, \\
& =x_{1}^{3}+9 x_{2}^{3}+81 x_{3}^{3}-27 x_{1} x_{2} x_{3}=1 .
\end{aligned}
$$

From (4.3) we obtain a solution for $s=0, n=3$, after calculating the necessary $A_{i}^{(v)}$ from the pre-period and period of the periodic Jacobi-Perron algorithm of the numbers $w, w^{2}, w=9^{1 / 3}$,

$$
x_{1}=649 ; \quad x_{2}=312 ; \quad x_{3}=150 ;
$$

and for the triple $\left(y_{1}, y_{2}, y_{3}\right), y_{k}=A_{3,4-k}\left(9 ; x_{1}, x_{2}, x_{3}\right), k=1,2,3$,

$$
y_{1}=1 ; \quad y_{2}=12 ; \quad y_{3}=-6 \text {. }
$$

5. The generalized Pellian equation of $k$-type. We shall introduce a combined sigma sign used by the author in a previous paper [1(f)], viz.

$$
\begin{aligned}
& \sum_{i=0 \mid c}^{t-1 \mid n} a_{i}=c \sum_{i=0}^{t-1} a_{i}+\sum_{i=t}^{n} a_{i}, \quad(t=1, \ldots, n) ; \\
& \sum_{i=0 \mid c}^{t-1 \mid n} a_{i}=\sum_{i=0}^{n} a_{i}, \quad \text { for } t<1 .
\end{aligned}
$$


In this chapter we shall prove

THEOREM 3. An infinite system of solutions of the generalized Pellian equation of $k$-type is given by the formula

$$
\begin{aligned}
x_{u}=\sum_{j=0 \mid d}^{k-u \mid n-u}\left(\begin{array}{c}
n-u \\
j
\end{array}\right) D^{j} A_{0}^{(s n+2 n-k+u-1+j)} ; \\
\quad(u=1, \ldots, n ; s=0,1, \ldots), k=1, \ldots, n-1 .
\end{aligned}
$$

The numbers $A_{0}^{(s n+2 n-k+u-1+j)}$ as defined by (3.7) are obtained from the periodic Jacobi-Perron algorithm of the numbers $w, w^{2}, \ldots, w^{n-1} ; w, D, d, n$ have the meaning of (3.10) and $d>1$.

Proof. We shall introduce the notation

$$
\begin{aligned}
& x_{u}^{(i)}=\sum_{j=0 \mid d}^{k-u \mid n-u}\left(\begin{array}{c}
n-u \\
j
\end{array}\right) D^{j} A_{i}^{(s n+2 n-k+u-1+j)}, \quad(s=0,1, \ldots) ; \\
& x_{u}^{(0)}=x_{u} ; \quad(u=1, \ldots, n ; i=0,1, \ldots, n-1), k=1, \ldots, n-1 .
\end{aligned}
$$

If we multiply each of the first $k$ column vectors of the determinant (3.8) by $d$ and substitute $s n+2 n-k$ for $v$ we obtain, since $(-1)^{(s n+2 n-k)(n-1)}=(-1)^{k(n-1)}$,

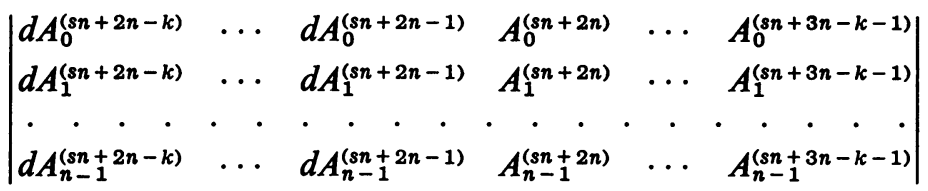

$$
\begin{aligned}
& =(-1)^{k(n-1)} d^{k} ; \quad k=1, \ldots, n-1 .
\end{aligned}
$$

If we add to the $i$ th column vector of the determinant (5.4) successively the $D\left(C_{n-i, 1}\right)$ multiple of the $i+1$ th vector, then the $D^{2}\left(C_{n-i, 2}\right)$ multiple of the $i+2$ th vector, $\ldots$, and finally the $D^{n-i}\left(C_{n-i, n-i}\right)$ multiple of the $i+(n-i)=n$th vector, $(i=1, \ldots, n-1)$ the determinant (5.4), with the notation of (5.3) takes the form

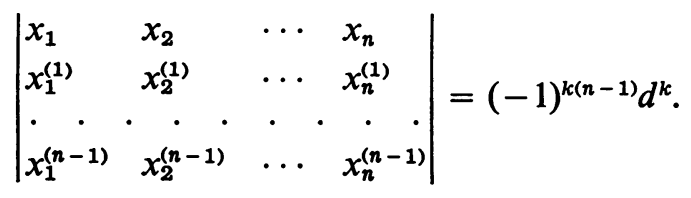

We shall again make use of formula (3.9) and obtain, for $v=s n+2 n-k$ and remembering that $a_{j}^{(s n+2 n-k)}=a_{j}^{(2 n-k)}$ for $k \leqq n ; j=1, \ldots, n-1$,

$$
a_{i}^{(0)}=\frac{A_{i}^{(s n+2 n-k)}+\sum_{j=1}^{n-1} a_{j}^{(2 n-k)} A_{i}^{(s n+2 n-k+j)}}{A_{0}^{(s n+2 n-k)}+\sum_{j=1}^{n-1} a_{j}^{(2 n-k)} A_{0}^{(s n+2 n-k+j)}} \quad(i=1, \ldots, n-1) .
$$


From (3.13) we obtain, substituting there $n-k$ for $k(n-k=1, \ldots, n-2)$

$$
\begin{aligned}
& a_{j}^{(2 n-k)}= \sum_{v=0}^{j}\left(\begin{array}{c}
n-1-j+v \\
v
\end{array}\right) w^{j-v} D^{v} \quad(j=1, \ldots, k-1), \\
& a_{k-1+s}^{(2 n-k)}=d^{-1} \sum_{v=0}^{k-1+s}\left(\begin{array}{c}
n-k-s+v \\
v
\end{array}\right) w^{k-1+s-v} D^{v} \\
&(s=1, \ldots, n-k), k=2, \ldots, n-1 .
\end{aligned}
$$

Since $a_{i}^{(2 n-1)}=a_{i}^{(n-1)}(i=1, \ldots, n-1)$ we obtain from (3.13)

$$
a_{i}^{(2 n-1)}=d^{-1} \sum_{j=0}^{i}\left(\begin{array}{c}
n-1-i+j \\
j
\end{array}\right) w^{i-j} D^{j} \quad(i=1, \ldots, n-1) .
$$

Substituting the values of $a_{j}^{(2 n-k)}(j=1, \ldots, n-1 ; k=1, \ldots, n-1)$ from (5.7) and (5.8) in (5.6) we obtain, arranging the numerator and denominator in powers of $w$, with the notation of (5.3) and substituting $w^{i}$ for $a_{i}^{(0)}(i=1, \ldots, n-1)$

$$
w^{i}=\frac{x_{1}^{(i)}+x_{2}^{(i)} w+x_{3}^{(i)} w^{2}+\cdots+x_{n}^{(i)} w^{n-1}}{x_{1}+x_{2} w+x_{3} w^{2}+\cdots+x_{n} w^{n-1}} \quad(i=1, \ldots, n-1) .
$$

From (5.9) we obtain, as in the previous chapter,

$$
\begin{gathered}
x_{q}^{(i)}=m x_{n-i+q} \quad(q=1, \ldots, i), \\
x_{i+j}^{(i)}=x_{j} \quad(j=1, \ldots, n-i), \\
i=1, \ldots, n-1 .
\end{gathered}
$$

Substituting the values of the $x_{r}^{(i)}(r=1, \ldots, n ; i=1, \ldots, n-1)$ from (5.10) in the determinant (5.5), we obtain

$$
D\left(m ; x_{1}, \ldots, x_{n}\right)=(-1)^{k(n-1)} d^{k} .
$$

With (5.11) and (5.3) Theorem 3 is completely proved. From Theorem 3 we immediately deduce

COROLlaRY 2. If the n-tuple $\left(x_{1}, x_{2}, \ldots, x_{n}\right)$ is a solution of the generalized Pellian equation of $k$-type, $D\left(m ; x_{1}, \ldots, x_{n}\right)=(-1)^{k(n-1)} d^{k}$, then the $n$-tuple $\left(y_{1}, y_{2}, \ldots, y_{n}\right), y_{k}=A_{n, n+1-k}(k=1, \ldots, n)$ is a solution of

$$
D\left(m ; y_{1}, \ldots, y_{n}\right)=(-1)^{k(n-1)} d^{k(n-1)}
$$

\section{Proof. From}

$$
\begin{aligned}
N\left(x_{1}+x_{2} w+x_{3} w^{2}\right. & \left.+\cdots+x_{n} w^{n-1}\right) \\
& =\left(x_{1}+x_{2} w+x_{3} w^{2}+\cdots+x_{n} w^{n-1}\right)\left(y_{1}+y_{2} w+y_{3} w^{2}+\cdots+y_{n} w^{n-1}\right) \\
& =(-1)^{k(n-1)} d^{k}
\end{aligned}
$$


we obtain

$$
\begin{aligned}
& N\left(x_{1}+x_{2} w+x_{3} w^{2}+\cdots+x_{n} w^{n-1}\right) N\left(y_{1}+y_{2} w+y_{3} w^{2}+\cdots+y_{n} w^{n-1}\right) \\
& =N\left((-1)^{k(n-1)} d^{k}\right)=(-1)^{k n(n-1)} d^{n k}=d^{n k}, \\
& N\left(y_{1}+y_{2} w+y_{3} w^{2}+\cdots+y_{n} w^{n-1}\right)=\frac{d^{n k}}{(-1)^{k(n-1)} d^{k}} \\
& =(-1)^{k(n-1)} d^{k(n-1)} \text {. }
\end{aligned}
$$

ExAmple. $n=3 ; D=d=2 ; m=2^{3}+2$; we choose $k=1$. The generalized Pellian equation of 1-type takes the form

$$
D\left(m ; x_{1}, x_{2}, x_{3}\right)=(-1)^{1 \cdot(3-1)} d=d ; \quad x_{1}^{3}+10 x_{2}^{3}+100 x_{3}^{3}-30 x_{1} x_{2} x_{3}=2 .
$$

From (5.3) we calculate for $s=0$

$$
x_{1}=15178 ; \quad x_{2}=7045 ; \quad x_{3}=3270 .
$$

A solution of the Diophantic equation

viz.

$$
D\left(m ; y_{1}, y_{2}, y_{3}\right)=(-1)^{k(n-1)} d^{k(n-1)},
$$

$$
y_{1}^{3}+10 y_{2}^{3}+100 y_{3}^{3}-30 y_{1} y_{2} y_{3}=4
$$

is given by

$$
y_{1}=184 ; \quad y_{2}=-10 ; \quad y_{3}=-35 \text {. }
$$

We shall now investigate the generalized Pellian equation of minus zero-type. On the basis of previous results obtained by the author [1(b), (f)] we shall first state the following

THEOREM. Write

$$
\begin{aligned}
w=\left(D^{n}-d\right)^{1 / n} ; \quad D, d, n \text { natural numbers } ; d \mid D ; \\
D \geqq 2(n-1) d ; \quad n \geqq 2 .
\end{aligned}
$$

The Jacobi-Perron algorithm of the $n-1$ numbers

$$
w, w^{2}, \ldots, w^{n-1}
$$

is periodic and the lengths of the pre-period and period are respectively

$$
\begin{array}{lll}
T=n^{2} ; & S=(n-1)^{2} & \text { for } d>1 ; \\
T=n ; & S=(n-1)^{2} & \text { for } d=1 .
\end{array}
$$

In case $d>1$ the $a_{i}^{\left(n^{2}\right)}$ have the form

$$
a_{i}^{\left(n^{2}\right)}=\sum_{j=0}^{i}\left(\begin{array}{c}
n-1-i+j \\
j
\end{array}\right) w^{i-j}(D-1)^{j} \quad(i=1, \ldots, n-1) .
$$


In case $d=1$ the $a_{i}^{\left(n^{2}-n\right)}$ also have the form (5.14)

$$
a_{i}^{\left(n^{2}-n\right)}=\sum_{j=0}^{i}\left(\begin{array}{c}
n-1-i+j \\
j
\end{array}\right) w^{i-j}(D-1)^{j} \quad(i=1, \ldots, n-1) .
$$

Comparing the $a_{i}^{\left(n^{2}\right)}$ and $a_{i}^{\left(n^{2}-n\right)}$ from (5.14), (5.15) with the $a_{i}^{(n)}$ from (3.8), we see that the only difference between the + zero-type and - zero-type cases of the generalized Pellian equation in this respect is that in the latter case $D-1$ has to be substituted for $D$. We can, therefore, state

THEOREM 4. An infinite system of solutions of the generalized Pellian equation $D\left(m ; x_{1}, \ldots, x_{n}\right)=1$ of minus zero-type is given by the formula

$$
x_{k}=\sum_{j=0}^{n-k}\left(\begin{array}{c}
n-k \\
j
\end{array}\right)(D-1)^{j} A_{0}^{\left(s n^{2}+k-1+j\right)} \quad(k=1, \ldots, n ; s=1,2, \ldots) .
$$

The numbers $A_{0}^{\left(s n^{2}+k-1+j\right)}$ as defined by (3.7) are obtained from the periodic JacobiPerron algorithm of the numbers $w, w^{2}, \ldots, w^{n-1} ; w, m, D, d, n$ have the meaning of (5.13) and $d>1$. For $d=1$ the same equation has an infinite system of solutions given by the formula

$$
x_{k}=\sum_{j=0}^{n-k}\left(\begin{array}{c}
n-k \\
j
\end{array}\right)(D-1)^{j} A_{0}^{(s n+k-1+j)} \quad(k=1, \ldots, n ; s \geqq n-1) .
$$

ExAmple. $n=3 ; D=2 ; d=1 ; m=2^{3}-1=7$. The generalized Pellian equation of minus zero-type takes the form

$$
x_{1}^{3}+7 x_{2}^{3}+49 x_{3}^{3}-21 x_{1} x_{2} x_{3}=1 .
$$

From (5.17) we calculate for $s=n-1$ with the $A_{i}^{(v)}$ from [1(b)]

$$
x_{1}=44 ; \quad x_{2}=23 ; \quad x_{3}=12 \text {. }
$$

From $w=\left(D^{n} \pm d\right)^{1 / n}, d, D, n$ as before, we deduce

$$
\begin{gathered}
\bar{w}=\left(D_{1}^{k} \pm d\right)^{1 / k} ; \quad \bar{w}=w^{n / k} ; \quad D_{1}=D^{n / k} ; k \mid n, k>1 ; \\
d \mid D_{1} ; \quad \bar{m}=D_{1}^{k} \pm d=m .
\end{gathered}
$$

The Jacobi-Perron algorithm of the numbers $\bar{w}, \bar{w}^{2}, \ldots, \bar{w}^{k-1}$ is periodic with length of pre-period $S=k-1$ and length of period $T=k$ or $T=1$. We can, therefore, formulate theorems for the solution of the generalized Pellian equation

$$
D\left(m ; x_{1}, \ldots, x_{k}\right)= \pm 1,
$$

analogous to Theorem 2 and Theorem 4, the essence of the new theorems being the fact that the solubility of $D\left(m ; x_{1}, \ldots, x_{n}\right)= \pm 1$ by means of a periodic Jacobi-Perron algorithm implies the solubility of $D\left(m ; x_{1}, \ldots, x_{k}\right)= \pm 1, k \mid n$. The units

$$
X_{e}=x_{1}+x_{2} \bar{w}+x_{3} \bar{w}^{2}+\cdots+x_{k} \bar{w}^{k-1}
$$

are in the ring $R(w)$ and create, therefore, possibly new units of the field $Q(w)$. 
6. Concluding remarks. Calculating, for $n=3$, the values of $y_{i}$ from (2.10) by means of the $x_{i}(i=1,2,3)$ from $(4.1)$ where we take $s=1$, we obtain easily

$$
y_{1}=1 ; \quad y_{2}=3 D^{2} / d ; \quad y_{3}=-3 D / d \text {. }
$$

Therefore, a unit in $Q\left(m^{1 / 3}\right), m=D^{3}+d, w=m^{1 / 3}$, has the form

$$
\begin{aligned}
Y_{e} & =1+\left(3 D^{2} / d\right) w-(3 D / d) w^{2}=\left(D^{3}+d-3 D w^{2}+3 D^{2} w-D^{3}\right) / d \\
& =\left(w^{3}-3 D w^{2}+3 D^{2} w-D^{3}\right) / d=(w-D)^{3} / d,
\end{aligned}
$$

and it is easy to prove by this method that a unit in $Q\left(m^{1 / n}\right), m=D^{n}+d, w=m^{1 / n}$, has the simple form

$$
Y_{e}=(w-D)^{n} / d .
$$

From what was said at the end of the last paragraph, other units of $Q\left(m^{1 / n}\right)$ are given by the formula

$$
Y_{e}=\left(w^{s}-D^{s}\right)^{n / s} / d ; \quad s \mid n ; \quad s<n .
$$

For $d=1$ these units have the form $Y_{e}=w^{s}-D^{s}, s \mid n, s<n$, and from the positive powers of $Y_{e}($ for $d \geqq 1)$ an explicit formula for the solutions of $D\left(m ; y_{1}, \ldots, y_{n}\right)$ $= \pm 1$ can be derived. It is equally easy to prove that (6.2) and (6.3) hold for the case $m=D^{n}-d$, too. These results were obtained, by quite a different approach to the problem, in a joint paper with Helmut Hasse [1(h)], my admired teacher, where we have developed a theory of units. In our paper a periodic algorithm of Jacobi-Perron was also the starting point of our investigations. In this context one is tempted to state that one of the central questions of number theory is not the calculation of units or the solution of generalized Pellian equations of \pm zero-type but the search for periodic algorithms (of Jacobi-Perron, or others; see the author's paper $[1(\mathrm{~m})]$ ) of algebraic numbers. This question is still challengingly open.

\section{REFERENCES}

LEON BERNSTEIN

1(a). Periodical continued fractions for irrationals of degree $n$ by Jacobi's algorithm, J. Reine Angew. Math. 213 (1963), 31-38.

1(b). Representation of $\left(D^{n}-d\right)^{1 / n}$ as a periodic continued fraction by Jacobi's algorithm, Math. Nachr. 1019 (1965), 179-200.

1(c). Periodicity of Jacobi's algorithm for a special type of cubic irrationals, J. Reine Angew. Math. 213 (1964), 134-146.

1(d). Periodische Jacobische Algorithmen für eine unendliche Klasse algebraischer Irrationalzahlen vom Grade $n$ und einige unendliche Klassen kubischer Irrationalzahlen, J. Reine Angew. Math. 214/215 (1964), 76-83.

1(e). Periodische Jacobi-Perronsche Algorithmen, Arch. Math. 15 (1964), 421-429.

1(f). New infinite classes of periodic Jacobi-Perron algorithms, Pacific J. Math. 16 (1966), 439-469.

1(g). A periodic Jacobi-Perron algorithm, Canad. J. Math. 17 (1965), 51-69.

LeON Bernstein and Helmut Hasse

1(h). Einheitenberechnung mittels des Jacobi-Perronschen Algorithmus, J. Reine Angew. Math. 218 (1965), 161-176. 
LEON BERNSTEIN

1(i). Rational approximations of algebraic irrationals by means of a modified Jacobi-Perron algorithm, Duke Math. J. 32 (1965), 161-176.

1(j). Periodische Kettenbrüche beliebiger Periodenlänge, Math. Z. 86 (1964), 128-135.

1(k). Rational approximation of algebraic numbers, Proc. Nat. Conf. on Data Processing, pp. 91-105, Rehovoth, Israel Proc. Assoc., 1964.

1(1). Explicit solution of an algebraic equation of degree $n$ by means of a modified JacobiPerron algorithm, SIAM Rev. (to appear).

1(m). The modified algorithm of Jacobi-Perron, its theory and application, Mem. Amer. Math. Soc. No. 67 (1967).

1(o). Der B-Algorithmus und seine Anwendung, J. Reine Angew. Math. (to appear).

1(p). Ein neuer Algorithmus Auer alsteigende Basispotenzen im Kubischen Koerper, Math. Nachr. Naas Feitschrift, 1967.

1(q). Periodic algorithms and units of algebraic fields, Hamburger Einzelhefte (to appear). C. G. J. JACOBI

2. Allgemeine Theorie der Kettenbruchähnlichen Algorithm, in welchen jede Zahl aus drei vorherfehenden gebildet wird, J. Reine Angew. Math. 69 (1868).

OsKar Perron

3. Grundlagen für eine Theorie des Jacobischen Kettenbruchalgorithmus, Math. Ann. 64 (1907).

Tel-Aviv University, Tel-Aviv, IsRael 